\title{
Research
}

\section{Are UK primary care teams formally identifying patients for palliative care before they die?}

\begin{abstract}
\section{Background}

The palliative care approach has the potential to improve care for patients with progressive lifethreatening illnesses from the time of diagnosis. Policy and clinical directives in the UK advocate early identification

Aim

To determine the extent to which practices identify patients for palliative care, including factors influencing early identification and possible effects on place of death.
\end{abstract}

\section{Design and setting}

Qualitative and quantitative data were collected from six general practices from three Scottish NHS boards and analysed.

\section{Method}

Records of patients who had died in the previous 6 months were analysed and interviews with practice staff ( $n=21)$ and with patients currently on the practice palliative care register and bereaved relatives ( $n=14)$ were conducted. In addition, a practice meeting was observed.

\section{Results}

In total, $29 \%$ of patients who died were recorded as being on the practice palliative care register before death. Two-thirds of patients with cancer were recorded on the register, but for those with non-malignant conditions only around $20 \%$ had any palliative care documented. This was a result of GPs not finding the current guidelines useful and being reluctant to discuss palliative care overtly with patients early in their illness. Palliative care services and documentation were geared towards patients with cancer. More district nurses than GPs saw the benefits of inclusion on the palliative care register. Only 25\% of patients on the register died in hospital.

\section{Conclusion}

Most patients with advanced progressive illnesses, especially those with non-malignant disease, are not being formally identified for a palliative care approach before they die. Those identified are more likely to benefit from coordinated care and may be more likely to die at home.

\section{Keywords}

family practice; palliative care; palliative care approach; primary care.

\section{INTRODUCTION}

The palliative care approach has great potential to support patients when they are diagnosed with any life-threatening progressive illness, not just in the last few days of life. 'Patients are known to have psychological and existential needs at diagnosis of advanced lung cancer and heart failure, and not just in their last days. ${ }^{2}$ In the US, a recent trial reported that early palliative care in patients with metastatic lung cancer increased their quality of life and patients lived longer. ${ }^{3}$

Internationally, GPs and district nurses have tended to identify patients later rather than earlier for palliative care, thereby avoiding difficult conversations, although most patients value an early opportunity to discuss their future. ${ }^{4}$

Clinicians rarely identify patients with non-malignant conditions for palliative care, except in the last days of life, although the needs of such patients can be as severe as, and often more prolonged, than those of someone with cancer. ${ }^{5}$

For over a decade in the UK, the Gold Standards Framework has called for more comprehensive and earlier identification of patients with progressive life-threatening diseases at practice level; this has been highlighted in recent English and Scottish government policy directives. ${ }^{6-8}$ Since 2006 , GP practices in the UK have been incentivised through the Quality and Outcomes Framework to keep a register of

N Harrison, MBChB, MSc (Public Health), research fellow; D Cavers, MSc, PhD, research fellow; C Campbell, BSc(Hons), PhD, MSc (Public Health), senior research fellow; SA Murray, MD, FRCGP, FRCP(Ed), St Columba's Hospice chair of primary palliative care, Primary Palliative Care Research Group, Centre for Population Health Sciences, University of Edinburgh, Edinburgh. Address for correspondence

Scott A Murray, Primary Palliative Care Research Group, Centre for Population Health Sciences, patients receiving palliative care and to hold regular multidisciplinary meetings to discuss these patients.?

In addition, since 2008, participating practices in Scotland have been further encouraged through the Scottish Palliative Care Directed Enhanced Services (DES) to identify patients as they enter the palliative care phase and, for each of these patients, to record a care plan, share this information with out-of-hours $(\mathrm{OOH})$ providers, and consider initiating an end-of-life pathway for the last days of life. ${ }^{10}$ In 2011 commissioners of end-of-life care were, advised to act early' to identify people 'approaching the end of their life when they are likely to die within the next 12 months. ${ }^{11}$ It is perceived, however, that these directives are only slowly being taken up in practice; ${ }^{12}$ as such, this study set out to:

- find out to what extent primary care teams in Scotland are currently identifying and documenting patients for a palliative care approach;

- identify the issues affecting this process; and

- explore whether there is an association between being identified for this approach and dying at home.

\section{METHOD}

\section{Setting and recruitment}

The study recruited six general practices -

University of Edinburgh, Edinburgh, EH8 9AG.

E-mail: Scott.Murrayded.ac.uk

Submitted: 4 August 2011; Editor's response: 8 November 2011; final acceptance:

1 December 2011

@British Journal of General Practice

This is the full-length article (published online $30 \mathrm{Apr}$ 2012) of an abridged version published in print. Cite this article as: Br J Gen Pract 2012; DOI: 10.3399/bjgp12X641465. 


\section{How this fits in}

Patients with malignant disease, and particularly those with non-malignant conditions, could benefit from better endof-life care but many are not identified for a palliative care approach. In this study, 29\% of patients were identified on practice palliative care registers before death; this included $68 \%$ of those dying of cancer and only $20 \%$ of those dying of non-malignant conditions. Patients on the practice palliative care register were more likely to die at home or in a hospice compared with those not on a register who tended to die in hospital. Challenges to early identification of such patients in primary care included GPs not seeing the benefits of it; being reluctant to talk to patients too early; not finding the guidelines helpful for systematic use; and primarily selecting patients with cancer.

two from each of three Scottish NHS boards - representing a range of practice sizes, urban and rural settings, and sociodemographic data. Six general practices were recruited through the local lead GP for palliative care in each of three Scottish NHS Boards.

\section{Data collection}

In each practice a mixed-methods approach was adopted. A retrospective analysis of all deaths in a recent 6-month period captured data on:

- date and place of death;

- primary diagnosis; and

- when, or if, patients were entered on the practice palliative care register before death.

Semi-structured interviews were conducted with GPs, district nurses, and practice administrators. The researcher observed a multidisciplinary palliative care meeting and had informal contact with other practice staff. Semi-structured interviews were also conducted with patients currently on the practice palliative care register and relatives of those who had been on the palliative care register and had died in the previous 4-12 months. Appendix 1 outlines the interviewees by practice.

\section{Analysis}

Interviews were digitally recorded, transcribed, and entered into NVivo 8 qualitative data analysis software IQSR
International). Thematic analysis began once the first interview had been transcribed and proceeded simultaneously with the data collection, both of which continued to inform the interview schedule. A thematic framework was developed by identifying key issues within the data using a priori research questions and emergent issues that were raised by interviewees. Contrasting themes and issues were examined with a view to developing explanations of participants' experiences and understandings, initially from different perspectives and then synthesised across all participants. ${ }^{13}$

\section{Definitions}

The following four categories described in the Gold Standards Framework ${ }^{14}$ were used to classify the deaths in each practice:

- 'sudden and unexpected' meant the patient had not been under regular care by the practice, or only for care such as blood-pressure checks, and had not been expected to die;

- 'cancer' was used if this was the major cause of death (remitted or quiescent cancers were not included if not contributory);

- 'dementia or physical frailty' included those with a clear record of progressive dementia and/or where frailty was recorded and there was no other specific diagnosis; and

- 'organ failure/chronic disease' included those recorded with progressive heart, respiratory, renal and/or liver failure, and those with other chronic progressive conditions.

'Place of death' was categorised into home, care home, hospice, community hospital, and hospital.

\section{RESULTS}

\section{Inclusion of patients on the palliative care} register

Proportion of patients who had recently died and were on the palliative care register. A total of 201 patients registered at the six practices died during the study period, of whom 59 (29\%) had been included on the practice palliative care register before death. The proportion varied considerably between practices (Table 1). Patients had been on the palliative care register for an average of 13 weeks before death (median 10 weeks) but the variance was large: from 2 days to 4 years. 
Table 1. Number of patients on the palliative care register before death, by practice

\begin{tabular}{|c|c|c|c|c|c|c|c|}
\hline & \multicolumn{6}{|c|}{ Practice } & \multirow[b]{2}{*}{ All practices } \\
\hline & 1 & 2 & 3 & 4 & 5 & 6 & \\
\hline Total deaths, $n$ & 52 & 29 & 17 & 43 & 40 & 20 & 201 \\
\hline Deaths on PCR, $n$ & 17 & 11 & 5 & 15 & 9 & 2 & 59 \\
\hline Proportion of all deaths on PCR, \% & 33 & 38 & 29 & 35 & 23 & 10 & 29 \\
\hline
\end{tabular}

Diagnoses of patients dying while on the palliative care register. Data were available on the cause of death for all but three of the 201 patients. Patients dying of cancer were much more likely to be included in the practice palliative care register before death compared with those with non-malignant conditions. A quarter of patients died suddenly and unexpectedly (Table 2).

\section{Factors influencing inclusion on the} palliative care register

The interviews and observation of practice team meetings revealed a number of themes critical to patients being included on the palliative care register.

Professionals' approach to identifying patients. Health professionals did not tend to use specific suggested guidance to identify patients eligible for inclusion on the palliative care register in a systematic way; instead they relied on a mixture of intuition, clinical knowledge, and subjective judgement. The most commonly mentioned trigger for identifying the patient for inclusion on the palliative care register was increased input from GPs and district nurses, in response to their deterioration in function and progression:

We know who our cancer patients are and we know the ones that we're needing to involve the district nurse so ... You know, largely speaking, it's when there's nurse involvement or team involvement that that's sort of palliative care ... I think it is very much when there's a team because it's about sharing information and making sure everything's done so it's very much when

\section{Table 2. Cause of death and inclusion on the palliative care register} before death

\begin{tabular}{lcc} 
Cause of death & $\begin{array}{c}\text { Patients with } \\
\text { diagnosis, } \boldsymbol{n}\end{array}$ & $\begin{array}{c}\text { Patients with } \\
\text { diagnosis on PCR, } \boldsymbol{n} \text { (\%) }\end{array}$ \\
\hline Cancer & 60 & 41 (68) \\
\hline Dementia/physical frailty & 39 & $8(21)$ \\
\hline Organ failure/chronic disease & 49 & $10(20)$ \\
\hline Sudden and unexpected & 50 & $\mathrm{~N} / \mathrm{A}$ \\
\hline Missing data & 3 & $\mathrm{~N} / \mathrm{A}$ \\
\hline All deaths in the six practices & 201 & $\mathbf{5 9}(\mathbf{2 9 )}$ \\
\hline N/A = not applicable. PCR palliative care register. & &
\end{tabular}

there's going to be somebody involved other than just me ... because then you're wanting the district nurse involved, you're maybe wanting other services and that's when the out-of-hours should know what's happening '(GP 4)

Some GPs found the 'surprise question' ('Would you be surprised if this patient were to die in the next 6-12 months?'), in particular, of limited help and over-inclusive, especially if the practice had a large population of older people: ${ }^{14}$

... you can see it happening and you can see people suddenly beginning to not function the way they should have been functioning. When it gets to heart failure ... dementia is a real hopeless one, and respiratory failure, I think if you did the "Do you expect this patient to be dead in a year", we're 100\% wrong ... All my patients have been on for over a year.' (GP 2)

Talking about end-of-life care. Some GPs would not add a patient to the palliative care register before they were fully aware of the patient's prognosis, whereas others were comfortable with a less-overt patient understanding. Many did not like to use the word 'palliative' with patients and found ways of working around this:

.. you may not actually say "You are on the palliative care register", but I do say to my patients, "The whole team is aware of what's going on, so you mustn't be frightened. You know, if there is a day, you know, I don't work and you need help, call, because the doctors and the nurses all know what's going on". So I may not call it a palliative care register, but they know that the team knows about them. (GP 8)

GPs acknowledged that they themselves might be reluctant to talk to patients about their terminal condition. They were concerned about destroying hope, even if they thought the patient probably already knew he or she was dying. It was easier to avoid this conversation for as long as possible, despite the possible consequences 
of not formally including the patient on the palliative care register:

And they were keeping up hope because they felt he needed it, and yet telling people they're dying when they all know they're dying, in one sense, isn't actually destroying hope, it's actually the professionals preserving themselves as well ... It's easier to keep on jollying people along sometimes than actually to sit down and have that very uncomfortable experience or even one conversation saying, "That's it, there's no point".' (GP 2)

\section{Clarity on the benefits of including a patient on the palliative care register}

GPs expressed uncertainty regarding the benefits to patients of being included on the palliative care register. They felt it did not change clinical care but did acknowledge that there were improvements in supportive aspects of care, especially with regard to information sharing and access to services. For example, patients would be discussed at the multidisciplinary meeting and the whole practice team became aware of their situation; they would have quicker phone access to GPs and district nurses, and a sensitive hearing at reception:

$\because$, and I'm sure my partners as well, tell the patients right up front, "Don't worry about appointments, phone anytime, you know, leave a message, we can phone you back relatives as well". So we try and take any barriers down that might have been there from before.' (GP 5)

In Scotland, when patients are included on the palliative care register, an electronic summary is sent to $\mathrm{OOH}$ services with the permission of the patient. GPs expressed additional concern that the information sent to $\mathrm{OOH}$ services was not being used. In one case, this resulted in an avoidable admission of a patient who died within hours of arrival:

I look at local out-of-hours sheets and, even on people for whom we know we sent in [a palliative care summary], they $[\mathrm{OOH}$ services] don't seem to have used that information sometimes to do what they want to do. So I'm not entirely convinced that the system works as we think it does ...' (GP 6)

Conversely, GPs who worked for $\mathrm{OOH}$ services were very positive about having this information available:

' experience it because I still do out of hours ... and that's the bit that's really useful is having information about people you went to see on somebody else's palliative care register and having a good chunk of information about them there.' (GP 1)

In contrast to GPs, district nurses saw more tangible patient benefits of the palliative care register: once included on the register a gateway opened to services such as specialist palliative care, carer support, sitter services, and priority access to other services, such as district nurse $\mathrm{OOH}$ services:

.... [Palliative care] community nursing is one of the kind of gateways to services for patients. It's not always about what we can supply but, quite often, what we know and how we can direct them to get other services. So we are an information system. Also things like equipment that can make life easier in the home, referral onto other agencies like social services, housing, social work, voluntary agencies. It's endless really. (District nurse 7)

Patients and relatives also spoke of the personalised care and improved access to the practice when on the palliative care register:

.... The receptionist got to know us, and I would phone up and say, "Oh it's about $\mathrm{Mr}$ $X^{\prime \prime}$, and they would always get somebody. And if I phoned again and said, "Look, I'm really, really worried", you know, you never felt that you were too much trouble, and somebody always came.' (Relative 2)

\section{Patients who did not have cancer}

Particular difficulties were reported in deciding when, and if, a patient who did not have cancer should be included on the palliative care register. Patients with, for instance, heart or respiratory failure might deteriorate markedly only to regain function, for example, after adjustment of medication or treatment of infection, often after admission to hospital; as such, a palliative phase was harder to define.

Similarly, for patients with dementia, who were often in care homes, good physical care had resulted in increased longevity and subsequent difficulty in predicting their death. The care of these patients may effectively be palliative in approach for several years, but GPs were reluctant for patients to be on the palliative care register for such a long time:

.... it's much more difficult with people who have more unpredictable diseases like 
Table 3. Place of death for patients and inclusion on the palliative care register before death

\begin{tabular}{|c|c|c|c|}
\hline & $\begin{array}{c}\text { All deaths } \\
\%(n)\end{array}$ & $\begin{array}{c}\text { Deaths on PCR } \\
\%(n)\end{array}$ & $\begin{array}{c}\text { Deaths not on PCR } \\
\qquad \%(n)\end{array}$ \\
\hline Home/care home & $89(45)$ & $29(49)$ & $60(43)$ \\
\hline Hospice & $12(6)$ & $12(20)$ & $0(0)$ \\
\hline Community hospitala & $9(5)$ & $3(5)$ & $6(4)$ \\
\hline Hospital & $88(44)$ & $15(25)$ & 73(53) \\
\hline Total ${ }^{\mathrm{b}}$ & 198 (100) & 59 (100) & $139(100)$ \\
\hline
\end{tabular}

COPD [chronic obstructive pulmonary disease] and heart failure and dementia because [for] people with malignant disease it tends to be very intensive when you're actually doing it but it's a short period and, to some extent, that's relatively easy to cope with. But delivering palliative care to people who are slowly dying of something like heart disease can be much more difficult, because it's very difficult to be very specific with them about what the future holds for them. You know, they might die in a month or they might die in 3,4 , or 5 years...' (GP 1)

\section{Emphasis on cancer diagnoses}

All patients on the cancer register were reviewed and considered for inclusion on the palliative care register at the practices multidisciplinary team meeting. Other disease registers, such as for heart failure, COPD, or dementia were not routinely used in this way:

'I think the problem is, we tend to see this as a cancer thing and it tends to get used as a cancer thing. And even though there may be a small handful of people who might well benefit from it or fit with it, we don't often tend to use it for that.' (GP 6)

\section{Emphasis on the needs of patients with cancer}

Health professionals felt the documentation, guidance, and specialist palliative care was designed primarily with the needs of patients with cancer in mind:

'I think / struggled with [the summary form] a little bit with my man who didn't have a diagnosis of cancer. Trying to work out whether it would show enough information was my main struggle with it, and he hadn't had any radiotherapy or chemotherapy so there's like boxes, swathes of boxes, that go unfilled, but I don't know if there was anything, I mean, maybe a box to just type something. I think it is quite geared towards cancer but, you know, there's space to put things but it just takes a wee bit more looking around.' (GP 11)

This meant that patients who did not have cancer did not necessarily fit in with the systems and, therefore, possibly did not receive such a good service at the end of life. District nurses felt that patients who did not have cancer were missing out because they weren't identified formally for palliative care:

'It does make a difference, though, to what care that can get if they are on the palliative care register. They would get free personal care, which they would get anyway, but ... we can organise it for these patients. You don't have to go through social work. We, as care managers, can organise that ... But it would mean that a non-cancer patient who has palliative care needs would get everything provided so much quicker than if they weren't on the register, as such, and classed as palliative care ... We can make sure they get the benefits they are entitled to. They get [an] out-of-hours number, an out-of-hours form would be completed.' (District nurse 5)

One GP felt that, ethically, patients expectations should not be raised if services were not available:

... especially for non-cancer patients, there aren't always [the] support mechanisms available [as] for cancer patients. It's all very well to say we should treat end-of-life care the same way for everyone but, the fact is, the services available are not the same for everyone. So you have to ensure that there is some benefit to the patient in being in this process.' (GP 7 )

Patients who did not have cancer were often identified at a very late stage - in the last days and weeks of life - with district nurses flagging up patients whom they had been visiting and whose condition had deteriorated. District nurses tended to look out for certain signs of deterioration such as:

Indications like being bed bound, not eating, not taking medications, thinking about ... reviewing their medications at that stage, withdrawing unnecessary interventions. These kinds of things' (District nurse 7).

Patients and bereaved relatives also accepted cancer as life limiting more readily than non-malignant conditions. The deterioration of patients who did not have cancer was generally less clear, making it difficult for them and their carers to accept their outlook: 
'But then sometimes there's you and you're saying .... Oh ... I wish they could do something for this cough", but they keep telling you there's nothing they can do. And then it's difficult - you can't accept it.' (Relative to patient 5 who did not have cancer)

\section{Place of patients' death}

Using the data on patients in the study practices who had died, place of death was examined in relation to inclusion on the palliative care register before death. Patients on the register before death were less likely to die in hospital: about a quarter of patients on the palliative care register died in hospital compared with around half of those not on the register (Table 3). Sixteen of the 18 deaths that were not sudden and occurred at home involved patients on the palliative care register.

All of the patients who died in a hospice were on the palliative care register before death and all had cancer. One practice was geographically very close to a hospice, which increased specialist palliative care input:

I don't know whether that's because of our proximity to [the hospice] - there's a lot of familiarity now with the hospice. People are very aware of it and family or friends and so they're quite clued up with what they can do and then if they are seeing a nurse from the hospice and the community ... The sort of barrier's not there anyway, they're already sort of used to it, seeing someone from it, and they'll discuss it.' (GP 3)

\section{Provenance}

Freely submitted; externally peer reviewed.

\section{Competing interests}

The authors have declared no competing interests.

\section{Ethical approval}

Ethical approval was obtained from the South East Scotland Research Ethics Committee (reference number: 09/S1102/69). The researcher obtained an honorary contract with the appropriate NHS board for each participating general practice.

\section{Acknowledgements}

The authors would like to thank all the staff of the participating general practices, the local GP palliative care leads, and the patients and carers who were interviewed. They would also like to thank Morag Edwards who transcribed the interviews.

\section{Discuss this article}

Contribute and read comments about this article on the Discussion Forum: http://www.rcgp.org.uk/bjgp-discuss for those with non-malignant conditions.

For patients who did not have cancer, the unpredictable course of their illness led to particular difficulties in deciding when to talk to them. Level of support, which was perceived as being less available for these patients, also made them less likely to be included on palliative care registers. Most patients on the register died at home or in a hospice; in comparison, more of those who were not identified as eligible for palliative care died in hospital.

\section{Strengths and limitations}

The six practices were diverse, but are likely to have been confident in their palliative care provision to agree to participation in the research. The views of self-selected practice staff interviewees may not be representative of other colleagues within the practice or across the UK. Practices were more likely to select bereaved relatives for interview whose loved ones had died at home. On the whole, these factors are likely to increase the identification of patients for palliative care, so barriers described may be even more evident in other practices.

The data presented here reflect where practices have documented a patient as being on the palliative care register using an approved framework. Extrapolation from national uptake data for the Scottish Palliative Care DES in the financial year 2009-2010, combined with data on deaths in Scotland, suggests that around $24 \%$ of people were on a palliative care register at death: close to the $29 \%$ found in this study (Appendix 2). It is recognised that other patients not on the palliative care register may have received holistic care that was entirely consistent with palliative care. One GP spoke about the similarity of care before and after a patient was put on the palliative care register - that particular practice's approach was holistic for all their patients with chronic illnesses.

It is unlikely that all patients, even excluding those whose deaths were sudden and unexpected, could be included on a palliative care register. For example, in this study, several patients newly diagnosed with cancer died before they left hospital and never returned to the care of their GP. The observation that patients on the palliative care register before death were more likely to die outside hospital cannot be interpreted as causative. Practices may select patients for the palliative care register whom they expect to care for at home. In addition, place-of-death data for home and care homes was combined as usual place of residence, but this may not be the case for 
patients who are admitted to care homes directly from hospital specifically for palliative care purposes.

\section{Comparison with existing literature}

In line with this study's findings, a comparison of patients dying of cardiorespiratory and malignant disease in two general practices in England found that patients with cancer were more likely to be recorded as being in receipt of palliative care, although both groups were equally likely to die at home. ${ }^{15}$ A qualitative exploration of the experiences of those dying with lung cancer and cardiac failure found that those with lung cancer were provided with more information about their illness and experienced a more predictable decline. ${ }^{16}$ Boyd et al also found that late recognition was common in general practice - there was a tendency for planning for dying, rather than planning for living and dying'. ${ }^{17}$ Practical advice on how to recognise when a patient might benefit from the supportive and palliative phase is available; ${ }^{18}$ this may help address the reluctance this study found clinicians have to introduce the subject of end-of-life care.

This study found a higher proportion (25\%) of sudden unexpected deaths than in previous estimates. The Gold Standards Framework Prognostic Indicator Guidance ${ }^{14}$ and the National Audit Office report of $2008^{19}$ outlined an estimated $5-10 \%$ sudden deaths, although GPs in the Gold Standards Framework's 2009 National Snapshot reported that $42 \%$ deaths were unexpected. ${ }^{20}$ This variance may, in part, be definitional or may be due to the population studied. Some of the difficulties of providing comprehensive palliative care in the $\mathrm{OOH}$ period in Scotland, previously identified, ${ }^{21}$ have been addressed by the national availability of summary palliative care information to $\mathrm{OOH}$ providers. GPs in this study who worked for $\mathrm{OOH}$ services found this summary information invaluable, but other GPs interviewed were not convinced it was being used fully. The proportion of patients dying in hospital in this study was similar to that previously reported. ${ }^{19,20}$

\section{Implications for practice and research}

Including more patients on the practice palliative care register increases coordination and communication, and has the potential to decrease the proportion of patients dying in hospital. This policy and practice initiative needs further support and continuing priority. The benefits of including patients on the practice palliative care register should be highlighted to practice teams to encourage them to do so.

There are differing views among health professionals regarding the appropriate time to include a patient on the palliative care register. District nurses want the opportunity to register patients before they become too ill, but GPs are reluctant for patients to be on the palliative care register for $>1$ year. Delayed identification of patients needing palliative care contributes to the low proportion identified, as patients may die suddenly. An intervention study addressing these barriers is needed so that more patients can be systematically identified.

Palliative care services and supporting documentation have often been designed with patients who have cancer in mind, such that other patients have to be 'fitted in' and services adapted to support them. ${ }^{22}$ End-oflife services should be reviewed and transformed to meet the needs of people dying on the three main trajectories of physical decline routinely, not just the more acute (typically cancer) trajectory. ${ }^{23}$ Practices also need to use pointers such as frequent $\mathrm{OOH}$ calls or hospital admissions, rather than a cancer diagnosis, to identify patients with potentially life-limiting conditions for inclusion on their register. Practices should also audit patient inclusion on the palliative care register, in addition to cause and place of death, and discuss results in multidisciplinary meetings. 


\section{REFERENCES}

1. World Health Organisation. WHO definition of palliative care. Geneva: WHO, 202. http://www.who.int/cancer/palliative/definition/en/ laccessed 6 Mar 2012).

2. Murray SA, Kendall M, Grant E, et al. Patterns of social psychological and spiritual decline towards the end of life in lung cancer and heart failure. J Pain Symptom Manage 2007; 34(4): 393-402.

3. Temel JS, Greer JA, Muzikansky A, et al. Early palliative care for patients with metastatic non-small-cell lung cancer. N Engl J Med 2010; 363(8): 733-742.

4. Davison SN, Simpson C. Hope and advance care planning in patients with end stage renal disease: qualitative interview study. BMJ 2006; DOI:10.1136/bmj.38965.626250.55.

5. Pinnock H, Kendall M, Murray SA, et al. Living and dying with severe chronic obstructive pulmonary disease: multi-perspective longitudinal qualitative study. BMJ 2011; 342: d142.

6. Gold Standard Framework. Shrewsbury: GSF. http://www.goldstandardsframework.org.uk/ laccessed 6 Mar 2012).

7. Department of Health. End of life care strategy - promoting high quality care for all adults at the end of life. London: Department of Health, 2008. http://www.dh.gov.uk/en/Publicationsandstatistics/Publications/PublicationsPo licyAndGuidance/DH_086277 (accessed 6 Mar 2012).

8. Scottish Government. Living and Dying Well: a national action plan for palliative and end of life care in Scotland. Edinburgh: The Scottish Government, 2008.

http://www.scotland.gov.uk/Publications/2008/10/01091608/0 laccessed 6 Mar 2012).

9. NHS Scotland. Revisions to the GMS contract for 2006/07. Delivering investment in general practice. Quality and Outcomes Framework 2006. Edinburgh: Health Department, 2006. http://www.sehd.scot.nhs.uk/pca/PCA2006(M)13.pdf laccessed 6 Mar 2012)

10. Scottish Palliative Care Directed Enhanced Service 2008. http://www.sehd.scot.nhs.uk/pca/PCA2008(M)12.pdf (accessed 6 Mar 2012).

11. The National Council for Palliative Care. Commissioning end of life care - act and early. London: NCPD, 2011.

12. Scottish Government. Living and dying well: building on progress. Edinburgh:
The Scottish Government, 2011.

http://www.scotland.gov.uk/Publications/2011/01/27090834/0 laccessed 6 Mar 2012).

13. Mason J. Qualitative researching. London: Sage Publications Ltd, 2002.

14. Gold Standards Framework. Prognostic indicator guidance. Shrewsbury: National Gold Standards Framework Centre, 2008.

http://www.goldstandardsframework.nhs.uk/Resources/Gold\%20Standards\% 20Framework/PDF\%20Documents/PrognosticIndicatorGuidancePaper.pdf laccessed 6 Mar 2012).

15. McKinley RK, Stokes T, Exley C, Field D. Care of people dying with malignant and cardiorespiratory disease in general practice. Br J Gen Pract 2004; 54(509): 909-913

16. Murray SA, Boyd K, Kendall M, et al. Dying of lung cancer or cardiac failure: prospective qualitative interview study of patients and their carers in the community. BMJ 2002; 325(7370): 929

17. Boyd K, Mason B, Kendall M, et al. Advance care planning for cancer patients in primary care: a feasibility study. Br J Gen Pract 2010; DOI: 1033999/bjgp10X544032.

18. Boyd K, Murray SA. Recognising and managing key transitions in end of life care. BMJ 2010; 341: c4863.

19. National Audit Office. End of life care 2008. London: National Audit Office. http://www.nao.org.uk/publications/0708/end_of_life_care.aspx laccessed 6 Mar 2012).

20. End of life in primary care: 2009 National snapshot - executive summary. http://www.goldstandardsframework.nhs.uk/Resources/Gold\%20Standards\% 20Framework/PDF\%20Documents/Nat\%20snapshot\%20of\%20EOLC\%20exec \%20summary.pdf (accessed 6 Mar 2012).

21. Worth A, Boyd K, Kendall M, et al. Out-of-hours palliative care: a qualitative study of cancer patients, carers and professionals. Br J Gen Pract 2006; 56(522): 6-13

22. Munday D, Dale J, Murray SA. Choice and place of death: individual preferences, uncertainty, and the availability of care. J R Soc Med 2007; 100(5): 211-215

23. Lynn J. Palliative care beyond cancer: reliable comfort and meaningfulness: making a difference campaign. BMJ 2008; 336(7650): 958-959. 


\section{Appendix 1. Interviewees by practice}

\begin{tabular}{|c|c|c|c|c|c|c|}
\hline Practice & GP & $\begin{array}{c}\text { District } \\
\text { nurse }\end{array}$ & $\begin{array}{c}\text { Administrative } \\
\text { staff }\end{array}$ & $\begin{array}{l}\text { Patient on } \\
\text { PCR }\end{array}$ & $\begin{array}{c}\text { Carer of patient } \\
\text { on PCR }\end{array}$ & $\begin{array}{c}\text { Bereaved } \\
\text { relative }\end{array}$ \\
\hline 1 & 1 & 2 (shared interview) & 1 & 2 & 1 & 1 \\
\hline 2 & 2 & 1 & 0 & 1 & 1 & 1 \\
\hline 3 & 2 & 1 & 1 & 1 & - & 1 \\
\hline 4 & 2 & 1 & 0 & 1 & 1 & 1 \\
\hline 5 & 2 & 1 & 1 & 0 & 0 & 1 \\
\hline 6 & 2 & 1 & 0 & 1 & - & 0 \\
\hline Total & 11 & 7 & 3 & 6 & 3 & 5 \\
\hline
\end{tabular}

$P C R=$ palliative care register.

\section{Appendix 2. Estimated \% of deaths on palliative care register based on the Scottish Palliative Care Directed Enhanced Services uptake data for all practices in Scotland}

This is a rough estimate for \% deceased patients on PCR before death and should be treated with caution for the following reasons:

- The numerator and denominator are from different time periods and different cohorts. The numerator is derived from the number of reported patients on the PCR for practices participating in the DES in 2009-2010 and is not necessarily indicative of those who are going to die over the same period. The denominator for deaths in Scotland is the General Register of Scotland figure for 2009, not the financial year 2009-2010.

- The number of patients on the PCR of all practices is extrapolated from those practices participating in the DES: non-participating practices may have fewer (or more) patients on the PCR.

- DES totals of patients for 2009-2010 may not be typical of past and future years because it is not known how long the patients spend on the PCR before they die. If there were a trend, say, to put patients on the PCR at an earlier stage of their illness in 2009-2010, then a lower proportion might die that year, affecting the proportion who were on the PCR before death.

\section{Estimated \% deaths on PCR based on DES uptake data 2009-2010}

Total patients on PCR 2009-2010 for the 68\% of practices that participated in the DES 8804

\begin{tabular}{ll}
\hline Extrapolated total patients on PCR 2009-2010 for 100\% practices & 12947
\end{tabular}

Deaths in Scotland 2009 (from GROS) 53856

Rough estimate of \% on PCR from these data

24

$D E S=$ Directed Enhanced Services. GROS = General Register of Scotland. $P C R=$ palliative care register. 